Electrochemotherapy is a new therapeutic option for patients with locally spread melanoma. It is based on the phenomenon of reversible electroporation, i.e. a transient increase in permeability of cell membranes under the influence of an appropriately modulated electric field. This allows multiplication of toxicity of a cytostatic agent entering the tumour cell. It is highly effective, especially in the palliative treatment of cancers located in the integument of the human body (skin and subcutaneous tissue). Available literature provides a mandate both for the application of this method in the aforementioned cases as well as for further work on its development.

This paper focuses on reviewing the literature concerning the use of electrochemotherapy in the treatment of melanoma.

Key words: electrochemotherapy, melanoma, bleomycin.

Contemp Oncol (Pozn) 2018; 22 (1): 8-13 DOI: https://doi.org/10.5114/wo.2018.74387

\section{Electrochemotherapy in the treatment of melanoma}

\author{
Mateusz Wichtowski ${ }^{1}$, Dawid Murawa²
}

${ }^{1}$ Oncological and General Surgery Ward I, Greater Poland Cancer Center, Poznan, Poland 2Department of General and Minimally Invasive Surgery, Poland Baptism Monument Hospital, Gniezno, Poland

\section{Introduction}

Tumours, irrespective of their histology, can cause metastases to the skin or subcutaneous tissue. Between $8 \%$ and $45 \%$ of patients diagnosed with melanoma experience skin metastases of which $22.7 \%$ are satellite and in-transit tumours; $50.2 \%$ are metastases in regional lymph nodes and $28.1 \%$ are distant metastases [1]. In the case of superficial lesions local therapies include: surgical intervention, radiotherapy, cryotherapy, laser therapy, RFA ablation, local chemotherapy, local immunotherapy with BCG, isolated limb perfusion and as a last resort in the case of limbs - amputation [2-7]. In addition to local treatment, systemic therapy is increasingly used. Progress in new targeted therapies has been tremendous in recent years [2, 7]. Despite this, some of the superficial lesions are initially unresectable, e.g. due to their size or location - e.g. face. Complications such as bleeding, infections, psychological aspects often require application of local therapies, even in stage 4 of the disease [8].

One of the newer therapies applied in metastatic melanoma is electrochemotherapy (ECT) which combines physical properties of electroporation using electric current with chemical properties of chemotherapeutics. In this method, by applying an electric current to the tissue, we induce a temporary increase in permeability of the cell membrane, thus enabling a free flow of large molecules into the cell, including cytostatics that at baseline are not transported to the cytosol. As a result, their potential toxicity increases considerably [9]. A flow chart presenting the procedure of electrochemotherapy is shown in Fig. 1.

Combinations of different cytostatic agents and electroporation have been studied in many pre-clinical studies and clinical trials. These studies concerned, inter alia: bleomycin, cisplatin, carboplatin, mitomycin-c, and cyclophosphamide [10-13]. The highest rate of cytotoxic enhancement after application of electroporation was reported for bleomycin, which increased in toxicity by up to 1000 times and for cisplatin - up to 80 times.

The first results concerning application of ECT with the use of intravenous bleomycin for melanoma were described by Rudolf et al. in 1995, and a year later Glass et al. described their first experience with ECT and bleomycin administered intratumourally $[11,14]$. Both studies showed similar results with ORR of $92 \%$. ECT based on cisplatin is equally effective; however fewer clinical data are available describing its use, mainly due to the necessity to administer this cytostatic agent locally - into the tumour, which in the case of disseminated skin lesions in one patient is often infeasible [12].

The ECT procedure is presented in detail in two papers: ESOPE (European Standard Operating Procedures of Electrochemotherapy) and SOP (Standard Operating Procedures) $[9,15]$. In the case of qualification for treatment of patients with small, single lesions ( $<7$ lesions and $<2 \mathrm{~cm}$ diameter of each lesion), both bleomycin and cisplatin can be used locally - intratumourally. The procedure starts with administration of a chemotherapeutic agent, and after 1 minute electroporation is performed. If the skin lesions in the pa- 


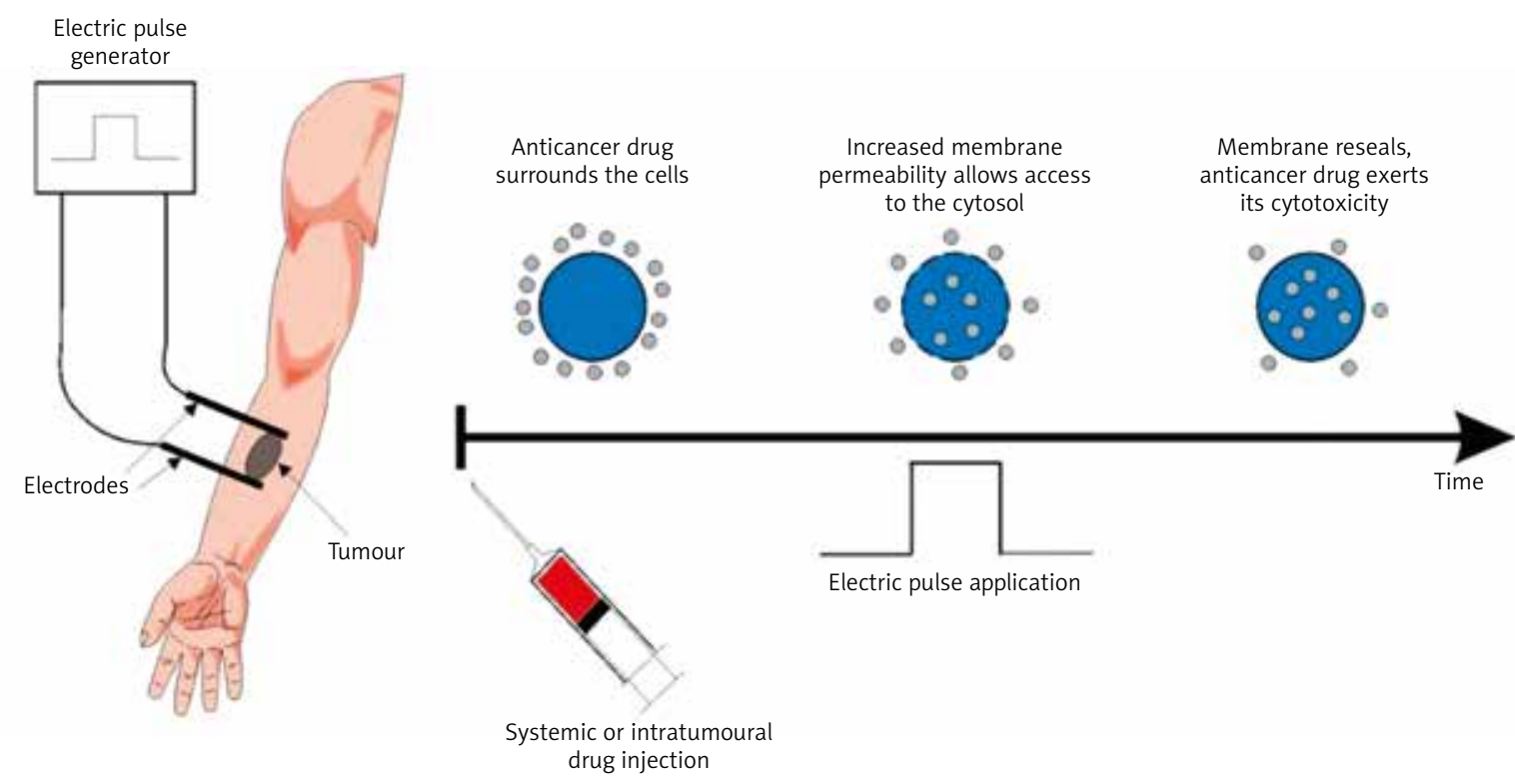

Fig. 1. Flow chart presenting steps in electrochemotherapy (by courtesy of IGEA Ltd.)

tient are above $2 \mathrm{~cm}$ in diameter or there are more than 7 lesions, bleomycin administered intravenously is the optimal cytostatic drug. Also in this case the procedure starts with administration of the cytostatic agent, and the supply of electric pulses begins after 8 minutes. The optimum concentration of the chemotherapeutic agent is maintained in the bloodstream from 8 to about 28 minutes after administration. Depending on the planned length of the procedure, it may be performed under local or general anaesthesia.

The ECT standards have been developed based on the use of a device called a Cliniporator (IGEA, Modena, Italy). This system provides several types of needle applicators that deliver current pulses to the tissue. The longest electrodes have a length of about $3 \mathrm{~cm}$, which is the maximum depth for an effective treatment of lesions present in the skin and subcutaneous tissue (Figs. 2, 3) [16].

The main contraindications to ECT include renal failure, allergy to bleomycin or cisplatin, pulmonary fibrosis (in the case of bleomycin), epilepsy, and a pacemaker. Nevertheless, according to the literature, ECT demonstrates a low toxicity profile and limited, mostly minor side effects. The main complications are local and include local pain, swelling, redness, ulcers (due to necrosis of originally exophytic tumours), and depigmentation $[9,16]$.

\section{Material and methods}

In the present paper, the method of electrochemotherapy was discussed and a review of literature was conducted concerning the treatment of melanoma with the use of ECT. This review included five databases: PubMed, HighWire Press, Science Direct, Wiley Online Library, and Google Scholar. Initially, 772 publications were selected by searching the phrases "electrochemotherapy" and "melanoma". We searched for publications on studies carried out after the publication of ESOPE standards, i.e. from
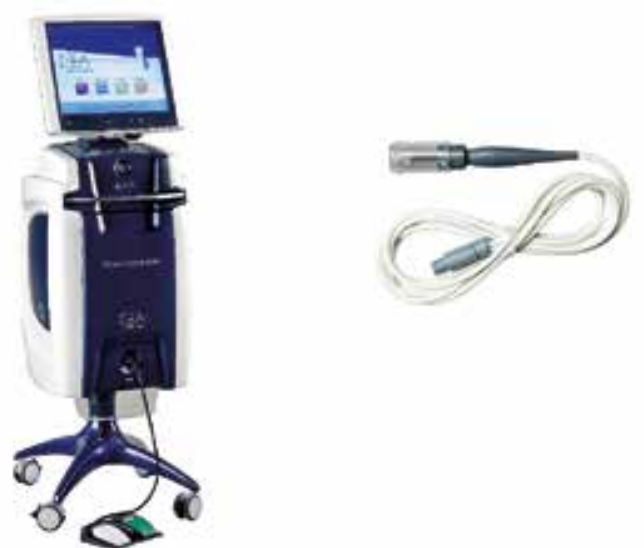

Fig. 2. Basic device Cliniporator ${ }^{\mathrm{TM}}$ with an applicator (by courtesy of IGEA Ltd.)

08.2006 until 07.2017. After a preliminary analysis we rejected the following: papers repeated in different databases, papers on preclinical studies, works only in the form of abstracts, publications in languages other than English or Polish, works in the form of presentations of clinical cases, reviews of literature and publications prepared on the basis of data from before the ESOPE study. We also excluded papers that described results of ECT on heterogeneous groups of patients, where a group of patients with melanoma was not clearly isolated. Ultimately, 11 original papers were qualified to the literature review. The scheme for selection of publications is presented in Fig. 4.

\section{Results}

The paper containing the latest standards in ECT in the treatment of metastatic melanoma is the aforementioned ESOPE [9]. The study included 61 patients from 4 European centres. ECT was performed using bleomycin i.t. or i.v. or cisplatin i.t. In this publication patients with melanoma 
A
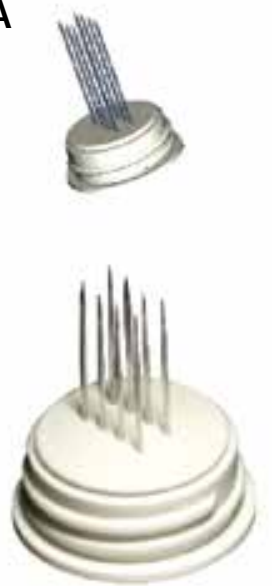

B
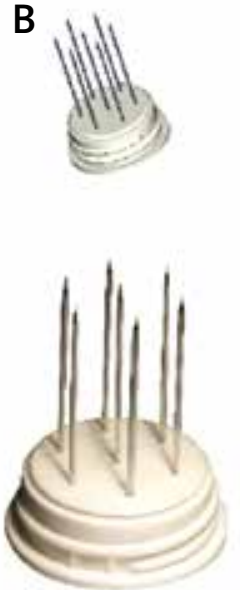

C
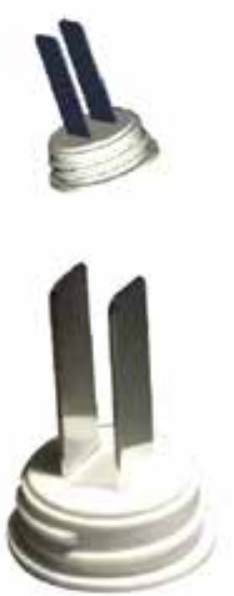

D
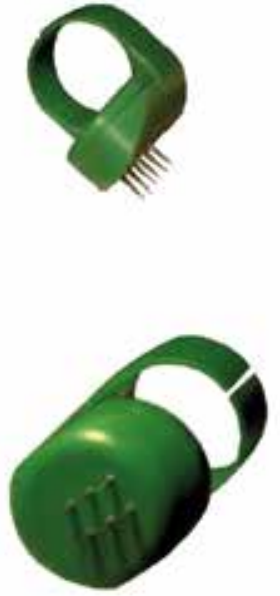

Fig. 3. Types of needle electrodes: A - linear; B - hexagonal; C - plate; D - ring (by courtesy of IGEA Ltd.)

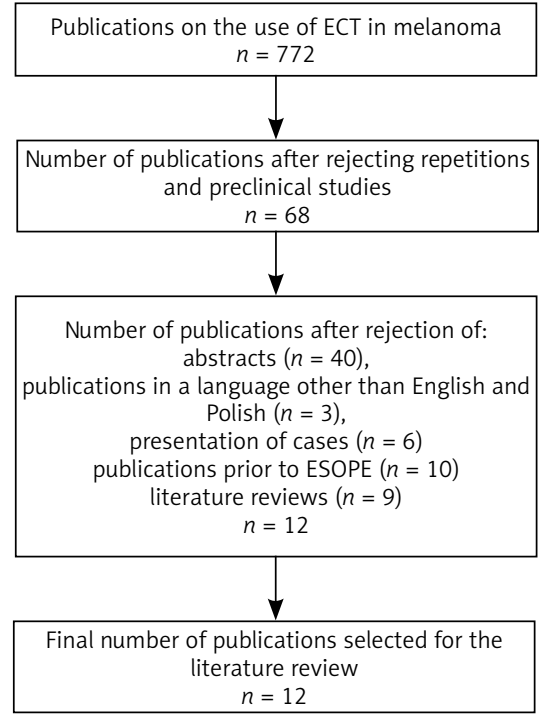

Fig. 4. The process of selection of publications included in the literature review

constituted the largest homogeneous group. In 20 patients included in the study ECT was performed on 99 skin lesions, achieving an OR (overall response) of $80.6 \%$ and CR (complete response) of $66.3 \%$.

One of the first works published after ESOPE was the paper by Gaudy et al. in which patients were randomised to two arms of the study - in the first arm the patients were treated with ECT using bleomycin administered intratumourally while in the other arm they received only bleomycin intratumourally [17]. The study ultimately gathered 40 patients, 24 of whom were randomised to the ECT group and 16 to the group with bleomycin alone. OR and CR for group 1 were $46 \%$ and 36\%, respectively, and $25 \%$ and $8 \%$ for group 2, respectively. Treatments were performed under local anaesthesia and the most common side effects were painful muscle spasms. Although a statistically significant difference was reported in CR, the group of patients with no response or progression of the disease dampened enthusiasm for this method because the group comprised $54 \%$ of all patients included in the study.

The best results with a representative group of $30 \mathrm{pa}$ tients and 654 treated skin lesions were published by Ricotti et al. in 2014 [18]. They evaluated CR for 20\% and PR (partial response) for $80 \%$ which together amounted to $100 \%$ of OR. A similar OR result was reported by Skarlatos et al.; however there were 5 patients with melanoma in this study [19].

A group of 60 patients was gathered by Caraco et al. who after 3 months of follow-up achieved OR of $86.6 \%$, and in $27.7 \%$ they achieved a CR to the treatment with a follow-up of at least 27.5 months [20].

A large group of patients with metastatic melanoma was treated with ECT by Campana et al. who achieved very good results. OR of $92 \%$ (CR 48\%, PR 44\%) [21]. After 2 years of follow-up LPFS (local progression-free survival) was $87 \%$, and after 26 months a local recurrence was observed in 6 patients from the originally CR group. The most common complications associated with the treatment included postoperative pain, vomiting, nausea, fever, and skin lesions (ulcers, discolouration).

Another paper presenting very good results was the publication of Quaglino et al. [22]. This paper described results of application of ECT on a group of 14 melanoma patients, and OR amounted to $93 \%$ after 8 weeks of follow-up. After 24 months of follow-up, $74.5 \%$ of CR patients had no local recurrence.

In 2014, Solari presented the results of his studies on ECT in melanoma [23]. In a group of 20 patients he used ECT with intravenous bleomycin and achieved CR and PR of $10 \%$ and $45 \%$, and SD (stable disease) and PD (progressive disease) in $15 \%$ and $30 \%$, respectively. It should be noted, however, that he used a longer follow-up period after which he assessed his patients, i.e. 6 months.

In 2017, Kunte and an international team published a study InspECT based on 151 patients demonstrating high efficacy of ECT [24]. The overall response was $73 \%$ with a minimum follow-up 60 days. One year overall survive was $67 \%$. The most common side effects were related to hyperpigmentation and ulceration (42\%, G3 in 2 patients), 
Table 1. Selected publications on the use of electrochemotherapy in the treatment of melanoma

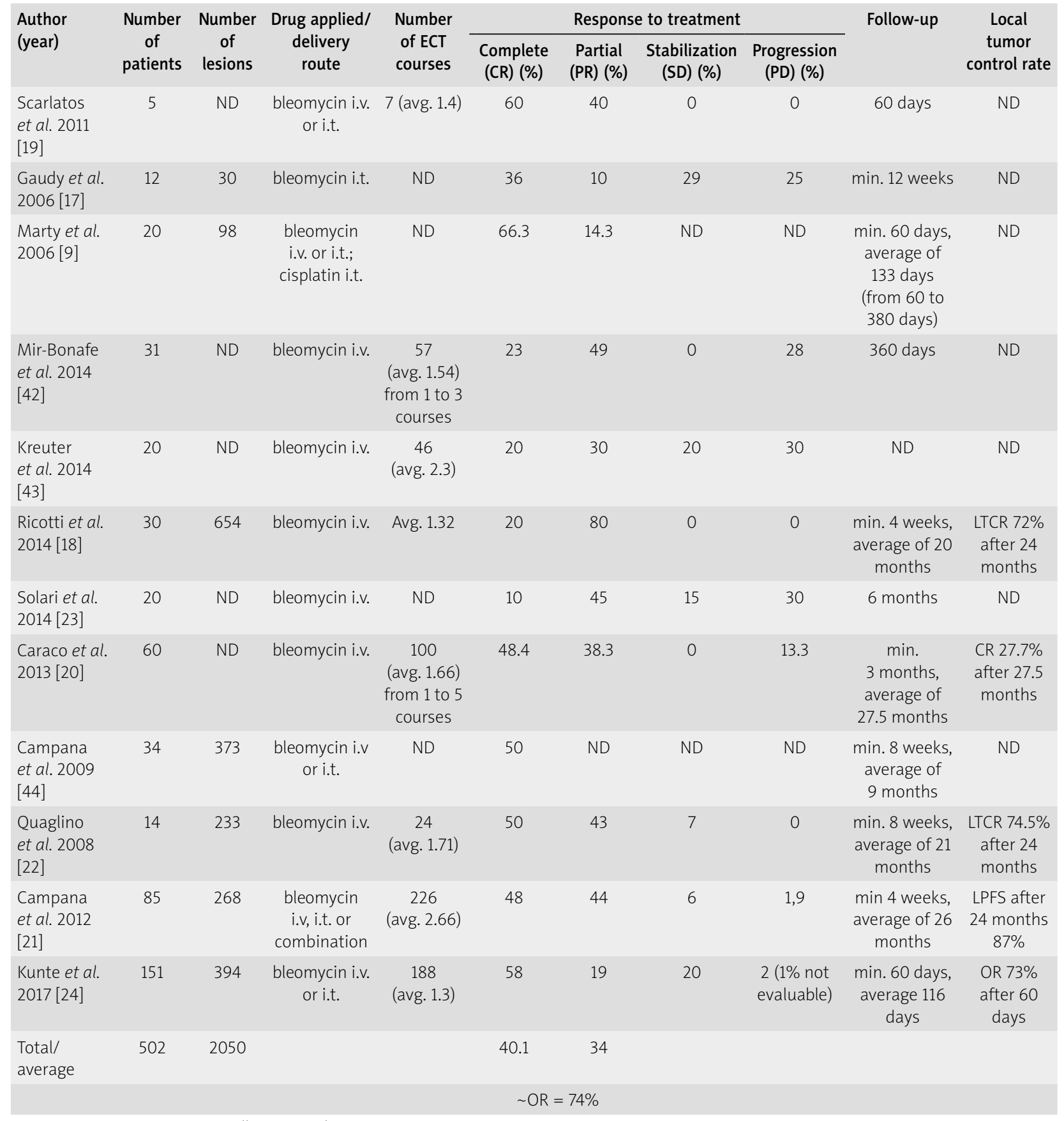

i.v. - intra venous; i.t. - intra tumorally; ND - no data

and others included: post-operative pain (39\%), flu-like symptoms (4\%), nausea (3\%), and swelling (3\%). This paper drew some important conclusions regarding ECT indications. In multivariate analysis, factors positively associated with overall response were tumour size $<3 \mathrm{~cm}$, absence of visceral metastases, treatment of non-irradiated areas, presence of lymphoedema, and coverage of deep margins and lateral margins. This publication was a prospective multicentre study on a large group of patients and therefore these results strongly confirm the conclusions of the previous publications and prove the effectiveness of the method.
All data collected from the publications included in the literature review are presented in Table 1.

\section{Conclusions}

In the case of limited in-transit melanoma metastases to the skin and subcutaneous tissue surgery remains the main therapeutic option. The most available and, therefore, most frequent intervention used in the case of cutaneous metastatic cancer in the case of ineligibility for surgery is a systemic chemotherapy and immunotherapy. Other methods used include radiotherapy and techniques 
of local therapies e.g. PDT (photodynamic therapy) and local chemotherapy (intralesional therapy - ILT). A meta-analysis published in 2014 presenting the results of 47 prospective studies compared five available therapies used for skin cancers (ECT, RT [radiotherapy], PDT, topical treatment - injection of a cytostatic drug into the tumour [ILT], and systemic treatment) - in the case of ECT the OR result was $75.4 \%$ (CR 47.5\%), with a low toxicity profile ( $\geq 3$ toxicity based on the CTC toxicity scale in less than $6 \%$ of patients). OR for RT was $62.7 \%$; OR for PDT was 67.8\%; OR for ILT was 21.4\%; and OR for systemic therapy amounted to $12.9 \%$ [25]. In the presented meta-analysis melanoma recurrence accounted for $83,3 \%$ of cases.

The efficacy of ECT has been proven in the treatment of many cancers, including melanoma, Kaposi's sarcoma, leiomyosarcoma, breast cancer spread, head and neck cancers, and primary skin cancers [19, 26-28]. Most often it is a palliative treatment when other methods do not bring a positive effect [29]. However, due to its proven efficacy, ECT can be considered a method of choice in the case of contraindications to surgery and radiotherapy, e.g. for a palliative provision of bleeding ulcers, thus improving the quality of life of patients. The advantage of ECT over other therapies is that it can be applied when chemotherapy, radiotherapy, and surgery have been used earlier and it can be repeated in the same area several times.

Different mechanisms are postulated to explain the achieved anti-tumour effect of ECT. The best documented include: an which increase in the intracellular concentration and, consequently, in activity of cytostatic drugs administered locally or systemically [30, 31]; induction of ischaemia in the mechanism of local contraction of arterioles (including the so called "vascular lock" - a delayed wash-out of the drug from the treated area) $[32,33]$ and the toxicity for endothelial cells [34, 35]; promotion of inflammatory infiltration in the treated area [36, 37]; toxicity associated with electrolysis and migration of electrolytes in the intracellular matrix and with disruptions of the transmembrane potential [38, 39].

An increasing number of studies showing the influence of ECT on immunological response were the basis of combining ECT with immunotherapy. Two recent studies seeking to evaluate the potency of the combination of ipilimum$\mathrm{ab}$ and ECT have produced very encouraging results [40, 41]. In the case report published by Brizio et al. [40], local ECT treatment of cutaneous lesions of melanoma was followed by ipilimumab administration, resulting in the complete regression of all the cutaneous and visceral metastases for at least 1 year. Interestingly, vitiligo-like lesions developed exclusively around the sites of previous ECT, suggesting that a prior ECT-driven immune activation was enhanced by ipilimumab. The other study reported that the volume of distant non-ECT-treated tumours decreased or was stabilised in nine patients out of 15 , possibly through ipilimumab-induced regulatory $T$ cell depletion [41].

In the near future we can expect further studies on new combinations of ECT with immunotherapy. Not only drugs but also nucleic acids can be transferred into target cells via the delivery of electroporation, e.g. electro-gene therapy, i.e. the electroporation-mediated transfer of therapeu- tic genes, which is currently under clinical evaluation and can potentially bring us to the next level of cancer fighting.

These methods, however, are relatively new and requires further studies, especially in large randomised groups of patients.

The authors declare no conflict of interest.

\section{References}

1. Meier F, Will S, Ellwanger U, Schlagenhauff B, Schittek B, Rassner G, Garbe C. Metastatic pathways and time courses in the orderly progression of cutaneous melanoma. Br J Dermatol 2002; 147: 62-70.

2. Eapen S, Dutcher JP. A review of evidence-based treatment of stage IIB to stage IV melanoma. Cancer Invest 2005; 23: 323-37.

3. Bonenkamp JJ, Thompson JF, de Wilt JH, Doubrovsky A, de Faria Lima R, Kam PC. Isolated limb infusion with fotemustine after dacarbazine chemosensitisation for inoperable loco-regional melanoma recurrence. Eur J Surg Oncol 2004; 30: 1107-12.

4. Lindner P, Doubrovsky A, Kam P, Thompson JF. Prognostic factors after isolated limb infusion with cytotoxic agents for melanoma. Ann Surg Oncol 2002; 9: 127-36.

5. McKay AJ, Byrne DS. Other treatments. In: Textbook of Melanoma. Thompson JF, Morton DL, Kroon BBR (eds.). Martin Dunitz, London 2004; 438-44.

6. Stevens G, Thompson JF, Firth I, O'Brien CJ, McCarthy WH, Quinn MJ. Locally advanced melanoma: results of postoperative hypofractionated radiation therapy. Cancer 2000; 88: 88-94.

7. Hersey P, Coates AS, McCarthy WH, et al. Adjuvant immunotherapy of patients with high-risk melanoma using vaccinia viral lysates of melanoma: results of a randomized trial. J Clin Oncol 2002; 20: 4181-90.

8. Balch CM, Buzaid AC, Atkins MB, et al. A new American Joint Committee on Cancer staging system for cutaneous melanoma. Cancer 2000; 88: 1484-91.

9. Marty M, Sersa G, Garbay JR, et al. Electrochemotherapy - An easy, highly effective and safe treatment of cutaneous and subcutaneous metastases: Results of ESOPE (European Standard Operating Procedures of Electrochemotherapy) study. EJC Suppl 2006; 4: 3-13.

10. Gehl J, Skovsgaard T, Mir LM. Enhancement of cytotoxicity by electropermeabilization: an improved method for screening drugs. Anticancer Drugs 1998; 9: 319-25.

11. Glass LF, Pepine ML, Fenske NA, Jaroszeski M, Reintgen DS, Heller R. Bleomycin-mediated electrochemotherapy of metastatic melanoma. Arch Dermatol 1996; 132: 1353-7.

12. Sersa G, Stabuc B, Cemazar M, Miklavcic D, Rudolf Z. Electrochemotherapy with cisplatin: clinical experience in malignant melanoma patients. Clin Cancer Res 2000; 6: 863-7.

13. Mir LM, Tounekti O, Orlowski S. Bleomycin: revival of an old drug. Gen Pharmacol 1996; 27: 745-8.

14. Rudolf Z, Stabuc B, Cemazar M, et al. Electrochemotherapy with bleomycin. The firms clinical experience in malignant melanoma patients. Radiol Oncol 1995; 29: 229-35.

15. Lluis M, Gehl J, Sersa G, et al. Standard operating procedures of the electrochemotherapy: instructions for the use of bleomycin or cisplatin administered either systemically or locally and electric pulses delivered by the Cliniporator by means of invasive or non-invasive electrodes. EJC Supplements 2006; 4: 14-25.

16. Kis E, Oláh J, Ócsai H, Baltas E, Gyulai R, Kemény L, Horvath AR. Electrochemotherapy of cutaneous metastases of melanoma a case series study and systematic review of the evidence. Dermatol Surg. 2011; 37: 1-9.

17. Gaudy C, Richard MA, Folchetti G, Bonerandi JJ, Grob JJ. Randomized controlled study of electrochemotherapy in the local treatment of skin metastases of melanoma. J Cutan Med Surg 2006; 10: 115-21. 
18. Ricotti E, Giuliodori K, Cataldi I, Campanati A, Ganzetti G, Ricotti G, Offidani A. Electrochemotherapy: an effective local treatment of cutaneous and subcutaneous melanoma metastases. Dermatologic Therapy 2014; 27: 148-52.

19. Scarlatos I, Kyrgias G, Mosa E, et al. Electrochemotherapy in can cer patients: first clinical trial in Greece. In Vivo 2011; 25: 265-74.

20. Caraco C, Mozzillo N, Marone $U$ et al. Long-lasting response to electrochemotherapy in melanoma patients with cutaneous metastasis. BMC Cancer. 2013; 13:564

21. Campana LG, Valpione S, Mocellin S, Sundararajan R, Granziera E, Sartore L, Chiarion-Sileni V, Rossi CR. Electrochemotherapy for disseminated superficial metastases from malignant melanoma. $\mathrm{Br}$ J Surg 2012; 99: 821-30.

22. Quaglino P, Mortera C, Osella-Abate S, Barberis M, Illengo M, Rissone M, Savoia P, Bernengo MG. Electrochemotherapy with intravenous bleomycin in the local treatment of skin melanoma metastases. Ann Surg Oncol 2008; 15: 2215-22.

23. Solari N, Spagnolo F, Ponte E, Quaglia A, Lillini R, Battista M, Queirolo P, Cafiero F. Electrochemotherapy for the management of cutaneous and subcutaneous metastasis: a series of 39 patient treated with palliative intent. J Surg Oncol 2014; 19: 270-4.

24. Kunte C, Letule V, Gehl J, et al. Electrochemmotherapy in the treatment of metastatic malignant melanoma: A prospective cohort study by InspECT. Br J Dermatol 2017; 176: 1475-85.

25. Spratt DE, Gordon Spratt EA, Wu S, DeRosa A, Lee NY, Lacouture ME, Barker CA. Efficiency of skin-directed therapy for cutaneous metastases from advanced cancer: a meta-analysis. J Clin Oncol 2014; 32: 3144-55.

26. Sersa G, Miklavcic D, Cemazar M, et al. Electrochemotherapy in treatment of tumours. Eur J Surg Oncol 2008; 34: 232-240.

27. Moller M, Salwa S, Soden D, et al. Electrochemotherapy as an adjunct or alternative to other treatments for unresectable or in-transit melanoma. Expert Rev Anticancer Ther 2009; 9: 1611-30.

28. Gehl J. Electroporation: theory and methods, perspectives for drug delivery, gene therapy and research. Acta Physiol Scand 2003; 177: 437-47.

29. Snoj M, Cemazar M, Slekovac Kolar B, et al. Effective treatment of multiple unresectable skin melanoma metastases by electrochemotherapy. Croat Med J 2007; 48: 391-5.

30. Mir LM, Orlowski S, Belehradek J, et al. Electrochemotherapy potentiation of antitumour effect of bleomycin by local electric pulses. Eur J Cancer 1991; 27: 68-72.

31. Jaroszeski MJ, Dang V, Pottinger C, Hickey J, Gilbert R, Heller R. Toxicity of anticancer agents mediated by electroporation in vitro. Anticancer Drugs 2000; 11: 201-8.

32. Sersa G, Cemazar M, Parkins CS, Chaplin DJ. Tumour blood flow changes induced by application of electric pulses. Eur J Cancer 1999; 35: 672-7.

33. Jarm T, Cemazar M, Miklavcic D, et al. Antivascular effects of electrochemotherapy: implications in treatment of bleeding metastases. Expert Rev Anticancer Ther 2010; 10: 729-46.

34. Cemazar M, Parkins CS, Holder AL, Chaplin DJ, Tozer GM, Sersa G. Electroporation of human microvascular endothelial cells: evidence for an anti-vascular mechanism of electrochemotherapy. $\mathrm{Br}$ J Cancer 2001; 84: 565-70.

35. Markelc B, Sersa G, Cemazar M. Differential Mechanisms Associated with Vascular Disrupting Action of Electrochemotherapy: In travital Microscopy on the Level of Single Normal and Tumor Blood Vessels. PLoS One 2013; 8: 1-11.

36. Orlowski S, An D, Belehradek J, et al. Antimetastatic effects of elec trochemotherapy and of histoincompatible interleukin-2-secreting cells in the murine Lewis lung tumor. Anticancer Drugs 1998; 9: 551-6.

37. Serša G, Miklavcic D, Cemazara M, Belehradek J, Jarm T, Mir LM. Electrochemotherapy with CDDP on LPB sarcoma: Comparison of the anti-tumor effectiveness in immunocompetent and immunodeficient mice. Bioelectrochemistry and Bioenergetics 1997; 43: 279-83

38. Nilsson E, von Euler $\mathrm{H}$, Berendson J, et al. Electrochemical treatment of tumours. Bioelectrochemistry 2000; 51: 1-11.

39. Turler A, Schaefer $H$, Schaefer $N$, Wagner M, Maintz D, Oiao JC, Hoelscher AH. Experimental low-level direct current therapy in liver metastases: influence of polarity and current dose. Bioelectromagnetics 2000; 21: 395-401.

40. Brizio M, Fava P, Astrua C, Cavaliere G, Savoia P. Complete regression of melanoma skin metastases after electrochemotherapy plus ipilimumab treatment: an unusual clinical presentation. Eur Dermatol 2015; 25: 271-2.

41. Mozzillo N, Simeone E, Benedetto L, Curvietto M, Giannarelli D, Gentilcore G, et al. Assessing a novel immuno-oncology-based combination therapy: ipilimumab plus electrochemotherapy. Oncoimmunology 2015; 4: e1008842.

42. Mir-Bonafe JM, Vilalta A, Alarcon I, et al. Electrochemotherapy in the Treatment of Melanoma Skin Metastases: a report on 31 cases. Actas Dermosifiliogr 2015; 106: 285-291.

43. Kreuter A, van Eijk T, Lehmann P, et al. Electrochemotherapy in advanced skin tumors and cutaneous metastases - a retrospective multicenter analysis. J Dtsch Dermatol Ges 2015; 13: 308-15.

44. Campana LG, Mocellin S, Basso M, et al. Bleomycin-based electrochemotherapy: clinical outcome from a single institution's experience with 52 patients. Ann Surg Oncol 2009; 16: 191-9.

\section{Address for correspondence}

\section{Dawid Murawa}

Department of General and Minimally Invasive Surgery

Poland Baptism Monument Hospital

3 Maja 37

62-200 Gniezno, Poland

e-mail:dmurawa@op.p

Submitted: 18.10.2017

Accepted: 16.12 .2017 